\title{
INTEGRATION OF ORDINARY LINEAR DIFFERENTIAL EQUATIONS BY LAPLACE-STIELTJES TRANSFORMS
}

\author{
BY
}

\section{JAMES D'ARCHANGELO AND PHILIP HARTMAN(1)}

ABSTRACT. Let $R$ be a constant $N \times N$ matrix and $g(t)$ an $N \times N$ matrix of functions representable as absolutely convergent Laplace-Stieltjes transforms for $t>0$. The paper gives sufficient conditions for certain solutions of the system $y^{\prime}=(R+g(t)) y$ to be expressed as Laplace-Stieltjes transforms or as linear combinations of such transforms with coefficients which are powers of $t$.

1. Introduction. This paper deals with $N$ th order linear differential equations

$$
D^{N} u+\sum_{j=0}^{N-1}\left[a_{j}+g_{j}(t)\right] D^{\prime} u=0, \quad \text { where } D u=d u / d t=u^{\prime} \text {, }
$$

and with first order systems of dimension $N$,

$$
y^{\prime}=[A+g(t)] y,
$$

where $a_{j}$ is a (complex) constant in (1.1) and $A$ is a (complex) constant $N \times N$ matrix in (1.2). In (1.1) or (1.2), we assume that $g_{j}(t)$ or $g(t)$ is representable as a Laplace-Stieltjes transform

$$
g_{j}(t)=\int_{0}^{\infty} e^{-s t} d G_{j}(s), \quad G_{j}(+0)=G_{j}(0)=0,
$$

or

$$
g(t)=\int_{0}^{\infty} e^{-s t} d G(s), \quad G(+0)=G(0)=0,
$$

absolutely convergent for $\operatorname{Re} t>0$ and satisfying

$$
\int_{+0} s^{-\gamma}\left|d G_{j}(s)\right|<\infty,
$$

or

$$
\int_{+0} s^{-\gamma}|d G(s)|<\infty,
$$

for a suitable constant $\gamma>0$. We consider the problem of the representation of certain solutions in terms of Laplace-Stieltjes transforms.

Received by the editors January 31, 1974. 44A10.

AMS (MOS) subject classifications (1970). Primary 34A25, 34A30, 34E05; Secondary

Key words and phrases. Laplace-Stieltjes transform, linear differential equations, Bessel and Legendre functions.

(1) The study was supported by National Science Foundation Grant GP-30483A2. 
First, our results can be interpreted as a generalization of the theory of regular singular points since the case where $g_{j}(t)$ or $g(t)$ are power series in $z=e^{-t}$ (i.e., $d G_{j}$ or $d G$ are atomic measures with support on the positive integers) corresponds to a regular singular point at $z=0$. Second, they provide sharpened variants of known results in the theory of asymptotic integrations. Finally, our results can be considered as a contribution to the theory of the representation of solutions in terms of definite integrals, a theory important to the study of solutions "in the large".

Our results imply the following theorem for the $N$ th order equation (1.1):

THEOREM 1.1. Let $P_{N}(\lambda)$ be the polynomial

$$
P_{N}(\lambda) \equiv \lambda^{N}+\sum_{j=0}^{N-1} a_{j} \lambda^{j}
$$

For a fixed real number $\tau$, let

$$
\{\lambda(1), \cdots, \lambda(K)\}=\text { set of distinct zeros of } P_{N}(\lambda): \operatorname{Im} \lambda=\tau,
$$

$$
\operatorname{Re} \lambda(1)<\cdots<\operatorname{Re} \lambda(K),
$$

$$
e(k)=\text { multiplicity of zero } \lambda=\lambda(k) \text { of } P_{N}(\lambda) \text {, }
$$

$$
E(k)=e(1)+\cdots+e(k) \text { and } E(0)=0 .
$$

Let the coefficient functions $g_{j}(t)$ be representable as Laplace-Stielties transforms (1.3), absolutely convergent for $\operatorname{Re} t>0$, satisfying (1.5) for

$$
\gamma \geqslant m(i), \text { where } m(i)=\max [e(1), \cdots, e(i)], .
$$

for some $i, 1 \leqslant i \leqslant K$. Finally, let $\delta>0$ be arbitrary if $i=1$, and $0<\delta<$ $\min [\lambda(2)-\lambda(1), \cdots, \lambda(i)-\lambda(i-1)]$ if $i \geqslant 1$. Then, for $\nu=0, \cdots, e(i)-1$, (1.1) has a solution $u=u(t)$ representable in the form

$$
u=e^{\lambda(i) t}\left\{t^{\nu}+t^{\nu} w(t)+t^{\nu+E(i-1)} w^{0}(t)\right\},
$$

where $w(t), w^{0}(t)$ are Laplace-Stielties transforms of the form

$$
w(t)=\int_{0}^{\delta} e^{-s t} d W(s), w(0)=W(+0)=0, w^{0}(t)=\int_{\delta}^{\infty} e^{-s t} d W^{0}(s),
$$

absolutely convergent for $\operatorname{Re} t>0$, and

$$
\int_{+0} s^{-\gamma+m(i)}|d W(s)|<\infty .
$$

The exponent $\nu+E(i-1)$ in (1.13) is suggested by the theory of regular singular points. Our results also imply the following theorem for the first order system (1.2).

THEOREM 1.2. Let the constant matrix $A$ be similar to the Jordan matrix $J=\operatorname{diag}[J(\lambda(1), e(1)), \cdots, J(\lambda(M), e(M))]$, where $J(\lambda, e)$ is an $e \times e$ Jordan 
block with $\lambda$ on the diagonal and 1 on the superdiagonal. For a fixed real number $\tau$, let

$$
\{\lambda(1), \cdots, \lambda(K)\}=\text { subset of }\{\lambda(k)\}: \operatorname{Im} \lambda(k)=\tau,
$$

$$
\operatorname{Re} \lambda(1)=\cdots=\operatorname{Re} \lambda\left(K_{1}\right)<\operatorname{Re} \lambda\left(K_{1}+1\right)=\cdots=\operatorname{Re} \lambda\left(K_{2}\right)<\cdots,
$$

where $K_{0}=0<K_{1}<\cdots<K_{h}=K$,

$$
\begin{aligned}
& e\left(K_{i}+1\right) \geqslant e\left(K_{i}+2\right) \geqslant \cdots \geqslant e\left(K_{i+1}\right) \quad \text { for } i=0, \cdots, h-1, \\
& E(i)=e(1)+e\left(K_{1}+1\right)+\cdots+e\left(K_{i-1}+1\right) \\
& \quad \text { for } i=1, \cdots, h \text { and } E(0)=0 .
\end{aligned}
$$

Let the $N \times N$ matrix $g(t)$ be representable as a Laplace-Stielties transform (1.4) absolutely convergent for $\operatorname{Re} t>0$, satisfying (1.5) for

$$
\gamma \geqslant m(i) \text {, where } m(i)=\max \left[e(1), \cdots, e\left(K_{i}\right)\right]
$$

for some $i, 1 \leqslant i \leqslant h$. Finally, let $\delta>0$ be arbitrary if $i=1$ and $0<\delta<$ $\min \left[\lambda\left(K_{2}\right)-\lambda\left(K_{1}\right), \cdots, \lambda\left(K_{i}\right)-\lambda\left(K_{i-1}\right)\right]$ if $i>1$. Then, for $K_{i-1}<n \leqslant$ $K_{i}$ and $\nu=0, \cdots, e(n)-1,(1.2)$ has a solution representable in the form

$$
y=e^{\lambda(n) t}\left\{\sum_{\mu=0}^{\nu} p_{\mu} t^{\mu}+t^{\nu} w(t)+t^{\nu+E(i-1)} w^{0}(t)\right\},
$$

where $w(t)$ and $w^{0}(t)$ are $N$-vectors representable as Laplace-Stielties transforms (1.14), absolutely convergent for $\operatorname{Re} t>0$ and satisfying (1.15), while $p_{\mu}$ is a constant vector and $p_{\nu} \neq 0$.

Theorem 1.1 is contained in Theorem 1.2 which in turn is implied by Theorem 2.1 below. For additional properties of the solutions (1.13) and (1.21) in Theorems 1.1 and 1.2, see Corollary 2.1 .

The simple case $A=0$ of Theorem 1.2 and certain cases of $N=2$ of Theorem 1.1 go back to Wintner, cf. [7] - [9]. For $i=1$, Theorems 1.1 and 1.2 are contained in sharper results of Hartman [3] and D'Archangelo [2], respectively. For $i>1$, D'Archangelo [2] gives results in which $t^{\nu} w+t^{\nu+E(i-1)} w^{0}$ of (1.13) and (1.21) is replaced by $t^{\nu} w$, where $w=w(t)$ is a Laplace-Stieltjes transform

$$
\begin{gathered}
w(t)=\int_{0}^{\infty} e^{-s t} d W(s), \quad W(0)=W(+0)=0, \\
\int_{+0} s^{-\gamma+m(i)}|d W(s)|<\infty
\end{gathered}
$$

These results (corresponding to the absence of certain "logarithmic terms" in the theory of regular singular points) are proved under assumptions including $d G_{j}(s)=0$ or $d G(s)=0$ for $0 \leqslant s \leqslant \lambda(i)-\lambda(1)$ or $0 \leqslant s \leqslant \lambda\left(K_{i}\right)-$ $\lambda(1)$. The proofs in [2], [3] involve successive approximations. In the 
first parts of the proofs, the convergence of the integrals for large $\operatorname{Re} t$ is easily established, but the second parts of the proofs for their convergence on $\operatorname{Re} t>0$ are more complicated and involve majorant equations or systems. Below, we prove the simple Lemma 2.1 which implies that actually the second parts of the proofs are not needed for the proofs of the statements of the theorems (in contrast to a statement concerning the convergence of sucessive approximations).

In §8, we apply Theorem 1.1 above (or rather Theorem 2.1 of [3]) to Bessel equations to obtain new integral representations for the Hankel functions $H_{\mu}^{(1)}, H_{\mu}^{(2)}$ similar to those of Whittaker [6]. When these are combined with the technique of $\S 11$ of [3], we obtain some apparently new functional relations for the Legendre functions.

2. Main theorem. In the first order system

$$
y^{\prime}=[J+g(t)] y
$$

of dimension

$$
N=e(1)+\cdots+e(M),
$$

let $J$ be a constant matrix in a Jordan normal form, say

$$
J=\operatorname{diag}[J(\lambda(1), e(1)), \cdots, J(\lambda(M), e(M))],
$$

where $J(\lambda, e)$ denotes an $e \times e$ Jordan block with $\lambda$ on the diagonal and 1 on the superdiagonal.

If $y$ is an $N$-vector, we write $y=\left(y_{1}, \cdots, y_{m}\right)$ where $y_{k}=\left(y_{k}^{0}, \cdots, y_{k}^{e(k)-1}\right)$ is an $e(k)$-vector. We call $y_{k}$ the $k$ th block of $y$ and $y_{k}^{\kappa}$ the $(k, \kappa)$ th component of $y$. In terms of components, (2.1) can be written

$$
y_{k}^{\kappa^{\prime}}=\lambda(k) y_{k}^{\kappa}+y_{k}^{\kappa+1}+\sum_{m=1}^{M} \sum_{\mu=0}^{e(m)-1} g_{k \mu}^{\kappa m}(t) y_{m}^{\mu}
$$

for $k=1, \cdots, M$ and $\kappa=0, \cdots, e(k)-1$, where $y_{k}^{e(k)}=0$, and $g_{k \mu}^{k m}(t)$ are the entries of the $N \times N$ matrix $g(t)$ with row index $(k, k)$ and column index $(m, \mu)$.

We shall let $1_{\kappa}^{k}$ denote the $N$-vector with $(k, \kappa)$ th component 1 and all other components 0. Put

$$
\xi_{\kappa}^{k}=\xi_{\kappa}^{k}(t)=\sum_{\mu=0}^{\kappa} 1_{\mu}^{k} t^{\kappa-\mu} /(\kappa-\mu) !
$$

so that $\xi_{k}^{k}$ is an $N$-vector, its $(m, \mu)$ th component is 0 if $m \neq k$ and its $k$ th block is $\left(t^{\kappa} / \kappa !, t^{\kappa-1} /(\kappa-1) !, \cdots, 1,0, \cdots, 0\right)$.

Assumption (A) In the system (2.1), i.e., (2.4), let the functions $g_{k \mu}^{\kappa m}(t)$ be representable as Laplace-Stieltjes transforms

$$
g_{k \mu}^{\kappa m}(t)=\int_{0}^{\infty} e^{-s t} d G_{k \mu}^{\kappa m}(s), \quad G_{k \mu}^{\kappa m}(0)=G_{k \mu}^{\kappa m}(+0)=0,
$$


absolutely convergent for $\operatorname{Re} t>0$. For a fixed real number $\tau$, let

$$
\{\lambda(1), \cdots, \lambda(K)\}=\text { set of eigenvalues of } J: \operatorname{Im} \lambda=\tau
$$

enumerated as follows

$$
\begin{aligned}
& \operatorname{Re} \lambda(1)=\cdots=\operatorname{Re} \lambda\left(K_{1}\right) \\
& <\operatorname{Re} \lambda\left(K_{1}+1\right)=\cdots=\operatorname{Re} \lambda\left(K_{2}\right)<\operatorname{Re} \lambda\left(K_{2}+1\right)=\cdots \\
& e\left(K_{j}+1\right) \geqslant e\left(K_{j}+2\right) \geqslant \cdots \geqslant e\left(K_{j+1}\right) \\
& \quad \text { for } j=0, \cdots, h-1 \text { and } K_{0}=0, K_{h}=K .
\end{aligned}
$$

We define

$$
E(i)=e(1)+e\left(K_{1}+1\right)+\cdots+e\left(K_{i-1}+1\right)
$$

and $i(k)$ is defined for $k=1, \cdots, K$ by

$$
K_{i(k)-1}<k \leqslant K_{i(k)}
$$

so that $1 \leqslant i(k) \leqslant h$.

THEOREM 2.1. Let Assumption (A) hold. Let $1 \leqslant i \leqslant h$ and assume that $\gamma(k) \geqslant 0$ for $k=1, \cdots, M$ and $\gamma(k) \geqslant e(k)$ for $k=1, \cdots, K_{i}$,

$$
\int_{+0} s^{-\gamma(k)}\left|d G_{k \mu}^{\kappa m}(s)\right|<\infty \text {. }
$$

Let $\delta_{i}>0$ be arbitrary if $i=1$ and

$$
0<\delta_{i}<\min \left[\lambda\left(K_{2}\right)-\lambda\left(K_{1}\right), \cdots, \lambda\left(K_{i}\right)-\lambda\left(K_{i-1}\right)\right]
$$

if $i>1$. Then, for $n=1, \cdots, K_{i}$ and $\nu=0, \cdots, e(n)-1$, there exists $a$ solution $y=y(t)$ of (2.1) of the form

$$
y(t)=e^{\lambda(n) t}\left\{\xi_{\nu}^{n}(t)+t^{\nu} w_{\nu}^{n}(t)+t^{\nu+E(i-1)} w_{\nu}^{n 0}(t)\right\},
$$

where the $(k, k)$ th components $w_{k \nu}^{k n}, w_{k \nu}^{k n 0}$ of the $N$-vectors $w_{\nu}^{n}, w_{\nu}^{n 0}$ are Laplace-Stieltjes transforms, absolutely convergent for $\operatorname{Re} t>0$, of the form

$$
w_{k \nu}^{k n}(t)=\int_{0}^{\delta i(n)} e^{-s t} d W_{k \nu}^{k n}(s) \text {, and } W_{k \nu}^{k n 0}(t)=\int_{\delta_{i(n)}-0}^{\infty} e^{-s t} d W_{k \nu}^{k n 0}(s) \text {, }
$$

such that $W_{k \nu}^{k n}(0)=W_{k \nu}^{k n}(+0)=0$,

$$
\begin{gathered}
\int_{+0} s^{-\gamma(k)+e(k)}\left|d W_{k \nu}^{k n}(s)\right|<\infty \text { for } 1 \leqslant k \leqslant K_{i(n)-1}, \\
\int_{+0} s^{-\gamma(k)+e(k)-\kappa}\left|d W_{k \nu}^{k n}(s)\right|<\infty \text { for } K_{i(n)-1}<k \leqslant K_{i(n)}, \\
\int_{+0} s^{-\gamma(k)}\left|d W_{k \nu}^{k n}(s)\right|<\infty \text { for } K_{i(n)}<k \leqslant M,
\end{gathered}
$$

for $\kappa=0, \cdots, e(k)-1$. 
REMARK. The proof will show that Theorem 2.1 remains valid if (2.12) is relaxed to

$$
\int_{+0} s^{-\gamma(k)+e(k)-\kappa-1}\left|d G_{k \mu}^{\kappa m}(s)\right|<\infty,
$$

for $1 \leqslant k \leqslant K_{1}$, while (2.12) is retained for $K_{1}<k \leqslant M$.

Unless the contrary is stated, it is assumed that in any Laplace-Stieltjes transform, say $x(t)=\int_{0}^{\infty} e^{-s t} d X(s)$, the determining function $X(s)$ is a complex valued function (or vector with complex valued components or matrix with complex valued entries) which is locally of bounded variation on $0 \leqslant s \leqslant \infty$, continuous from the right, and satisfies $X(0)=X(+0)=0$.

Below we consider the determining functions $W_{k \nu}^{k n}(s)$ and $W_{k \nu}^{k n 0}(s)$ in (2.14) to be defined for $0 \leqslant s<\infty$, where $W_{k \nu}^{k n}(s)=W_{k \nu}^{k n}\left(\delta_{i(n)}-0\right)$ for $s \geqslant \delta_{i(n)}$ and $W_{k \nu}^{k n 0}(s)=0$ for $0 \leqslant s<\delta_{i(n)}$. It will be seen that arguments of [3] and the proof of Theorem 2.1 imply

Corollary 2.1. Assume the conditions of Theorem 2.1.

(i) If $0<\Delta<\delta_{i(n)}$ and the functions $G_{k \mu}^{k m}(s)$ in (2.6) satisfy $d G_{k \mu}^{k m}(s)=0$ for $0 \leqslant s<\Delta\left(<\delta_{i(n)}\right)$ when $m=1, \cdots, K_{i(n)-1}$ and $\mu=$ $0, \cdots, e(m)-1$, or $m=n$ and $\mu=0, \cdots, \nu$ and arbitrary $(k, k)$, then $d W_{k \nu}^{k n}(s)=0$ for $0 \leqslant s<\Delta$ for all $(k, \kappa, n, v)$.

(ii) The discontinuities of the functions $W_{k \nu}^{k n}(s)$ are contained in the additive semigroup generated by the discontinuities of $G(s)=\left(G_{k \mu}^{k m}(s)\right)$.

(iii) If $G(s)$ is continuous [or absolutely continuous or of class $\left.C^{1}([0, \infty))\right]$, then $W_{k \nu}^{k n}(s)$ is continuous [or absolutely continuous or of class $C^{1}((0, \infty))$ ].

(iv) If $K_{1}<n \leqslant K$, then $W_{k \nu}^{k n 0}(s)$ is absolutely continuous on $s \geqslant 0$ except possibly for a discontinuity at $s=\delta_{i(n)}$.

In proving Theorem 2.1 , it suffices to show that (2.1) has a solution $y(t)$ of the form (2.13)-(2.17), where the Laplace-Stieltjes transforms $w_{k \nu}^{k n 0}(t)$ are absolutely convergent for large $\operatorname{Re} t$. Its absolute convergence for $\operatorname{Re} t>0$ is then implied by the following lemma and its corollary.

LEMMA 2 .1. Let $B$ be a constant $N \times N$ matrix, $h(t)$ an $N \times N$ matrix function representable as a Laplace-Stieltjes transform

$$
h(t)=\int_{0}^{\infty} e^{-s t} d H(s), \quad H(0)=H(+0)=0,
$$

absolutely convergent for $\operatorname{Re} t>0$. Let $z(t)$ be a solution of

$$
z^{\prime}=[B+h(t)] z
$$

representable as a Laplace-Stieltjes transform 


$$
z(t)=z(\infty)+\int_{0}^{\infty} e^{-s t} d Z(s), \quad Z(0)=Z(+0)=0,
$$

absolutely convergent for large $\operatorname{Re} t$, where $z(\infty)$ is a constant vector. Then the integral in (2.21) is absolutely convergent for $\operatorname{Re} t>0$.

The change of variables

$$
z=y e^{-\lambda(n) t} t^{-\nu-E}, \text { where } E=E(i(n)-1),
$$

in (2.1) transforms (2.13) to

$$
z(t)=\xi_{\nu}^{n}(t) / t^{\nu+E}+w_{\nu}^{n}(t) / t^{E}+w_{\nu}^{n 0}(t)
$$

and (2.1) into (2.20), where

$$
B=J-\lambda(n) I \text { and } h(t)=g(t)-(\nu+E) I / t .
$$

Since for $\operatorname{Re} \alpha>0$,

$$
t^{-\alpha}=\int_{0}^{\infty} e^{-s t} s^{\alpha-1} d s / \Gamma(\alpha)=\int_{0}^{\infty} e^{-s t} d\left[s^{\alpha} / \Gamma(1+\alpha)\right]
$$

is absolutely convergent for $\operatorname{Re} t>0$, the first two terms on the right of (2.23), up to an additive constant, and $h(t)$ in (2.24) are Laplace-Stieltjes transforms, absolutely convergent for $\operatorname{Re} t>0$. Thus Lemma 2.1 has the following consequence.

Corollary 2.2. Let Assumption (A) hold and let (2.1) have a solution of the form (2.13)-(2.14), where the integrals in (2.14) are absolutely convergent for large $\operatorname{Re} t$ [or more generally of the form

$$
y(t)=e^{\lambda(n) t}\left\{\xi_{\nu}^{n}(t)+t^{\nu+E} w_{\nu}^{n 1}(t)\right\},
$$

where $E \geqslant 0$ and $w_{\nu}^{n 1}(t)$ is representable as a Laplace-Stieltjes transform

$$
w_{\nu}^{n 1}(t)=\int_{0}^{\infty} e^{-s t} d W_{\nu}^{n 1}(s)
$$

absolutely convergent for large $\operatorname{Re} t$ ]. Then the integrals in (2.14) [or in (2.27)] are absolutely convergent for $\operatorname{Re} t>0$.

3. Notation and preliminaries. In addition to the conventions introduced above that, for any Laplace-Stieltjes transform

$$
x(t)=\int_{0}^{\infty} e^{-s t} d X(s),
$$

the determining function $X(s)$ is locally of bounded variation on $s \geqslant 0$ and satisfies $X(0)=X(+0)=0$, we always assume that (3.1) is absolutely convergent on some half-plane $\operatorname{Re} t \geqslant$ const. We shall say that

(3.2) $\gamma \geqslant 0$ is admissible for $x(t)$ or $X(s)$ if $\int_{+0} s^{-\gamma}|d X(s)|<\infty$. 
Proposition 3.1. Let $x(t), y(t)$ be representable as Laplace-Stielties transforms

$$
x(t)=\int_{0}^{\infty} e^{-s t} d X(s), \quad y(t)=\int_{0}^{\infty} e^{-s t} d Y(s)
$$

absolutely convergent for $\operatorname{Re} t \geqslant T$. Then the product $x(t) y(t)$ is representable as a Laplace-Stielties transform

$$
x(t) y(t)=\int_{0}^{\infty} e^{-s t} d Z(s), \text { where } Z(t)=\int_{0}^{t} X(t-s) d Y(s),
$$

absolutely convergent for $\operatorname{Re} t \geqslant T$. Furthermore,

$$
\delta \geqslant 0, \epsilon \geqslant 0, \quad \int_{+0} s^{-\delta}|d X(s)|<\infty, \text { and } \int_{+0} s^{-\epsilon}|d Y(s)|<\infty
$$

$$
\Rightarrow \int_{+0} s^{-\delta-\epsilon}|d Z(s)|<\infty
$$

Proof. The first part of this proposition is standard. Only the implication (3.5) has to be verified. Suppose first that $X$ and $Y$ have continuous derivatives, so that $Z$ has the continuous derivative

$$
Z^{\prime}(t)=\int_{0}^{t} X^{\prime}(t-s) Y^{\prime}(s) d s
$$

Thus we have

$$
\int_{0}^{1} s^{-\delta-\epsilon}|d Z(s)| \leqslant \int_{0}^{1} s^{-\delta-\epsilon} \int_{0}^{s}\left|X^{\prime}(s-r)\right|\left|Y^{\prime}(r)\right| d r d s
$$

and by Fubini's theorem, the last expression is

$$
\begin{aligned}
\int_{0}^{1}\left(\int_{r}^{1} s^{-\delta-\epsilon}\left|X^{\prime}(s-r)\right| d s\right)\left|Y^{\prime}(r)\right| d r \\
\leqslant \int_{0}^{1} r^{-\epsilon}\left\{\int_{r}^{1}(s-r)^{-\delta}\left|X^{\prime}(s-r)\right| d s\right\}\left|Y^{\prime}(r)\right| d r
\end{aligned}
$$

Consequently

$$
\int_{0}^{1} s^{-\delta-\epsilon}|d Z(s)| \leqslant\left(\int_{0}^{1} r^{-\epsilon}|d Y(r)|\right)\left(\int_{0}^{1} s^{-\delta}|d X(s)|\right)<\infty,
$$

and so (3.5) holds. It is clear that this inequality holds without any differentiability assumptions on $X, Y$.

Proposition 3.2. Let (3.1) be absolutely convergent for large $\operatorname{Re} t$. Then there exists a $T$ such that $[1+x(t)]^{-1}$ is representable as a LaplaceStieltjes transform

$$
[1+x(t)]^{-1}=1+\int_{0}^{\infty} e^{-s t} d X_{0}(s)
$$


absolutely convergent for $\operatorname{Re} t \geqslant T$. Also

$$
\delta \geqslant 0, \quad \int_{+0} s^{-\delta}|d X(s)|<\infty \Rightarrow \int_{+0} s^{-\delta}\left|d X_{0}(s)\right|<\infty .
$$

Proof. Let $T>0$ be large, so that $1+x(t+T)=1+\int_{0}^{\infty} e^{-s t} e^{-s T} d X(s)$ is absolutely convergent for $\operatorname{Re} t \geqslant 0$. Formally,

$$
[1+x(t+T)]^{-1}=\sum_{k=0}^{\infty}[-x(t+T)]^{k}=1+\int_{0}^{\infty} e^{-s t} d \sum_{k=1}^{\infty}(-1)^{k} X^{(k)}(s),
$$

where $d X^{(k)}(s)$ is the measure $\left(e^{-s T} d X(s)\right) * \cdots *\left(e^{-s T} d X(s)\right)$ obtained by convoluting $e^{-s T} d X(s)$ with itself $k$ times. Since $\int_{0}^{\infty}\left|d X^{(k)}(s)\right| \leqslant\left(\int_{0}^{\infty} e^{-s T}|d X(s)|\right)^{k}$, it can be supposed that $T$ is so large that the total variation $\int_{0}^{\infty} e^{-s T}|d X(s)|<1$, so that the first part of Proposition 3.2 follows.

In order to verify (3.8), note that by (3.6),

$$
\int_{+0}^{1} s^{-\delta}\left|d X^{(k)}(s)\right| \leqslant\left(\int_{0}^{1} s^{-\delta} e^{-s T}|d X(s)|\right)\left(\int_{0}^{\infty} e^{-s T}|d X(s)|\right)^{k-1}
$$

for $k \geqslant 1$. In view of (3.9), this gives (3.8) and completes the proof.

Proposition 3.3. Let $w=\left(w_{k m}(t)\right)$, where $k, m=1, \cdots, P$, be a matrix of Laplace-Stieltjes transforms such that $\sigma_{k} \geqslant 0$ is admissible for the off-diagonal elements $w_{k m}(t), m \neq k$, in the kth row. Let $Y=I_{P}+w$, where $I_{P}$ is the unit $P$-matrix. Then $Y=Y(t)$ is nonsingular for large $\operatorname{Re} t$ and $Y^{-1}=I_{P}+\bar{w}$, where $\bar{w}=\left(\bar{w}_{k m}(t)\right)$ is a matrix of Laplace-Stieltjes transforms, and $\sigma_{k}$ is admissible for the off-diagonal elements $\bar{w}_{k m}, m \neq$ $k$, in the kth row.

Proof. It follows from Propositions 3.1 and 3.2 that $[\operatorname{det} Y(t)]^{-1}=$ $1+x(t)$, where $x(t)$ is a Laplace-Stieltjes transform, and that $Y^{-1}=I_{P}+\bar{w}$, where $\bar{w}$ is a matrix of Laplace-Stieltjes transforms.

Note that if $k \neq m$, then $\bar{w}_{k m}=(-1)^{k+m}(1+x) x_{m k}$, where $x_{m k}$ is the cofactor of $w_{m k}$ in $Y$. Also, in the expansion of the determinant $x_{m k}$, each term contains an off-diagonal element $w_{k n}, n \neq k$, of the $k$ th row. Hence, $\sigma_{k}$ is admissible for each term in this expansion and, consequently, for $\bar{w}_{k m}$, by Proposition 3.1.

In order to state the next lemma, we introduce some notation. Let $x(t)$ be the Laplace-Stieltjes transform (3.1), absolutely convergent for $\operatorname{Re} t \geqslant T \geqslant$ 0 , and let $\Delta \geqslant 0$. Put

$$
x_{j \Delta}=\int_{0}^{\infty} e^{-s t} d X_{j \Delta}(s),
$$

where $X_{j \Delta}(s)$ is absolutely continuous for $s \geqslant 0$ and 
(3.11) $d X_{j \Delta}(s) / d s=e^{s T} \int_{0}^{s} e^{-r t}(s-r)^{j}(\Delta-r)^{-j-1} d X(r) / j !$ for $0 \leqslant s<\Delta$, $d X_{j \Delta}(s) / d s=(-1)^{j+1} e^{s T} \int_{s}^{\infty} e^{-r T}(r-s)^{j}(r-\Delta)^{-j-1} d X(r) / j ! \quad$ for $\Delta<s<\infty$.

It is easy to verify that (3.10) is absolutely convergent for $\operatorname{Re} t \geqslant T$. Note that the jump of $X(s)$ at $s=\Delta$, if any, does not contribute to (3.10), (3.11).

Proposition 3.4. Let $x(t)$ in (3.1) be a Laplace-Stielties transform absolutely convergent for $\operatorname{Re} t \geqslant T \geqslant 0, k \geqslant 0$ an integer, and $\Delta \geqslant 0$. Then

$$
\begin{array}{rl}
\int t & t(t-s)^{k} e^{\Delta s} x(s) d s / k ! \\
& =e^{\Delta t}(t-T)^{k+1} x_{k \Delta}(t)+c_{\Delta}(t-T)^{k+1} /(k+1) !
\end{array}
$$

where $c_{\Delta}=X(\Delta)-X(\Delta-0)$ is the jump of $X(s)$ at $s=\Delta$ (so that $c_{\Delta}=0$ if $\Delta=0)$.

Proof. It is clear that the last term in (3.12) is the contribution of the jump of $X(s)$ at $s=\Delta$ to the left side of (3.12). We can therefore suppose that $c_{\Delta}=0$ (i.e., that $X(s)$ is continuous at $s=\Delta$ ).

In order to prove (3.12) with $c_{\Delta}=0$, we first suppose that $d X(s) \equiv 0$ for small $|s-\Delta|, s \geqslant 0$. If $I$ is the left side of (3.12), then

$$
I=\int_{0}^{\infty}\left[\int_{T}^{t}(t-s)^{k} e^{(\Delta-r) s} d s / k !\right] d X(r)
$$

The inner integral is

$$
\begin{aligned}
{[\cdots]=} & -\sum_{j=0}^{k}(t-T)^{k-j} e^{(\Delta-r) T} /(k-j) !(\Delta-r)^{j+1} \\
& +e^{(\Delta-r) t /(\Delta-r)^{k+1} .}
\end{aligned}
$$

Hence, we have

$$
I=-\sum_{j=0}^{k}(t-T)^{k-j} \int_{0}^{\infty} e^{(\Delta-r) T}(\Delta-r)^{-j-1} d X(r) /(k-j) !+J_{1}+J_{2},
$$

where

$$
\begin{aligned}
& J_{1}=e^{\Delta t} \int_{0}^{\Delta} e^{-r(t-T)} e^{-r T}(\Delta-r)^{-k-1} d X(r), \\
& J_{2}=e^{\Delta t} \int_{\Delta}^{\infty} e^{-r(t-T)} e^{-r T}(\Delta-r)^{-k-1} d X(r) .
\end{aligned}
$$

Apply integration by parts to $J_{1}$ to obtain

$$
\begin{aligned}
J_{1}= & \int_{0}^{\Delta} e^{(\Delta-r) T}(\Delta-r)^{-k-1} d X(r) \\
& +e^{\Delta t}(t-T) \int_{0}^{\Delta} e^{-r(t-T)}\left[\int_{0}^{r} e^{-s T}(\Delta-s)^{-k-1} d X(s)\right] d r .
\end{aligned}
$$

Repeated integration by parts gives 


$$
\begin{aligned}
J_{1}= & \sum_{j=0}^{k}(t-T)^{k-j} \int_{0}^{\Delta} e^{(\Delta-r) T}(\Delta-r)^{-j-1} d X(r) /(k-j) ! \\
& +e^{\Delta t}(t-T)^{k+1} \int_{0}^{\Delta} e^{-r t} d X_{k \Delta}(r) .
\end{aligned}
$$

Similarly

$$
\begin{aligned}
J_{2}= & \sum_{j=0}^{k}(t-T)^{k-j} \int_{\Delta}^{\infty} e^{(\Delta-r) T}(\Delta-r)^{-j-1} d X(r) /(k-j) ! \\
& +e^{\Delta t}(t-T)^{k+1} \int_{\Delta}^{\infty} e^{-r t} d X_{k \Delta}(r) .
\end{aligned}
$$

Thus (3.12) follows from (3.15) and the last two displays, provided that $d X(s) \equiv 0$ for small $|s-\Delta|, s \geqslant 0$. The general case follows by approximating $X(s)$ suitably.

REMARK . Note that if $d X(s)=0$ for $0 \leqslant s \leqslant a$, then $d X_{k \Delta}(s)=0$ for $0 \leqslant s \leqslant a$. This fact will be used in proving the following:

Proposition 3.5. Let $x(t)$ in (3.1) be a Laplace-Stieltjes transform, absolutely convergent for $\operatorname{Re} t \geqslant T \geqslant 0, k \geqslant 0$ an integer, and $0<\Delta^{\prime}<\Delta$. Then

$$
\begin{aligned}
& \int_{T}^{t} s^{k} e^{\Delta s} x(s) d s / k ! \\
&= e^{\Delta t} \sum_{m=0}^{k} t^{k-m}(-1)^{m} \int_{0}^{\Delta^{\prime}} e^{-r t}(\Delta-r)^{-m-1} d X(r) /(k-m) ! \\
&+e^{\Delta t} t^{k+1} x_{k T \Delta \Delta^{\prime}}(t)
\end{aligned}
$$

where $x_{k T \Delta \Delta^{\prime}}(t)$ is a Laplace-Stieltjes transform with determining function satisfying

$$
d X_{k T \Delta \Delta^{\prime}}(s)=0 \text { for } 0 \leqslant s \leqslant \Delta^{\prime} .
$$

In particular, if $\gamma$ is admissible for $x(t)$, then $\gamma$ is admissible for the coefficient of $t^{k-m}$ in (3.16).

Proof. Write $x(t)=x_{0}(t)+x^{0}(t)$, where

$$
x_{0}(t)=\int_{0}^{\Delta^{\prime}} e^{-s t} d X(s) \text { and } x^{0}(t)=\int_{\Delta^{\prime}}^{\infty} e^{-s t} d X(s) .
$$

Then, by Fubini's theorem, we have

$$
\begin{aligned}
\int_{T}^{t} s^{k} e^{\Delta s} x_{0}(s) d s / k ! \\
\quad=e^{\Delta t} \sum_{m=0}^{k} t^{k-m}(-1)^{m} \int_{0}^{\Delta^{\prime}} e^{-r t}(\Delta-r)^{-m-1} d X(r) /(k-m) !+c,
\end{aligned}
$$

where $c=c\left(T, \Delta^{\prime}, \Delta\right)$ is a constant. If we write $c=e^{\Delta t} t^{k+1}\left(c e^{-\Delta t} / t^{k+1}\right)$, 
where $c e^{-\Delta t} / t^{k+1}$ is a Laplace-Stieltjes transform with a determining function vanishing for $0 \leqslant s<\Delta$, we see that the proposition is correct if $d X(s)=0$ for $s \geqslant \Delta^{\prime}$.

In order to deal with $x^{0}(t)$, write

$$
s^{k} / k !=[t-(t-s)]^{k} / k !=\sum(t-s)^{m}(-1)^{m} t^{k-m} / m !(k-m) !
$$

and use Proposition 3.4 to get

$$
\begin{aligned}
& \int_{T}^{t} s^{k} e^{\Delta s} x^{0}(s) d s / k ! \\
&= e^{\Delta t} \sum_{m=0}^{k} t^{k-m}(-1)^{m}(t-T)^{m+1} x_{m \Delta}^{0} /(k-m) ! \\
&+c_{\Delta}\left(t^{k+1}-T^{k+1}\right) /(k+1) !
\end{aligned}
$$

where $x_{m \Delta}^{0}$ belongs to $x^{0}$ as $x_{m \Delta}$ belongs to $x$. By the remark preceding this proposition, $d X_{m \Delta}^{0}(s)=0$ for $0 \leqslant s \leqslant \Delta^{\prime}$. The right side of (3.18) can be written as $e^{\Delta t} t^{k+1}$ times a Laplace-Stieltjes transform with determining function which vanishes for $0 \leqslant s \leqslant \Delta^{\prime}$. This completes the proof.

Proposition 3.6. Under the conditions of Proposition 3.5, there exist Laplace-Stielties transforms, absolutely convergent for $\operatorname{Re} t \geqslant T>0$,

$$
p(t)=\int_{0}^{\Delta^{\prime}} e^{-s t} d P(s), \quad p^{0}(t)=\int_{\Delta^{\prime}}^{\infty} e^{-s t} d P^{0}(s)
$$

such that if $\gamma$ is admissible for $x(t)$, then $\gamma$ is admissible for $p(t)$ and

$$
\int_{T}^{t} s^{k} e^{\Delta s} x(s) d s / k !=e^{\Delta t}\left[t^{k} p(t)+t^{k+1} p^{0}(t)\right]
$$

Proof. Define $P(t)$ by

$$
\sum_{m=0}^{k} t^{-m}(-1)^{m} \int_{0}^{\Delta^{\prime}} e^{-r t}(\Delta-r)^{-m-1} d X(r) /(k-m) !=\int_{0}^{\infty} e^{-s t} d P(s)
$$

and $P^{0}(t)$ by

$$
d P^{0}(s)=0 \text { or } d P^{0}(s)=d X_{k T \Delta \Delta^{\prime}}(s)+d P(s)
$$

according as $0 \leqslant s \leqslant \Delta^{\prime}$ or $\Delta^{\prime}<s$.

4. Proof of Lemma 2.1. In this section, in contrast to the remainder of the paper, we allow determining functions to have discontinuities at $s=0$. Thus we can write $h(t)$ in place of $B+h(t)$, and (2.19), (2.21) become

$$
h(t)=\int_{0}^{\infty} e^{-s t} d H(s) \text {, where } H(0)=0, H(+0)=B,
$$




$$
z(t)=\int_{0}^{\infty} e^{-s t} d Z(s) \text {, where } Z(0)=0, Z(+0)=z(\infty),
$$

while the differential equation (2.20) now has the form

$$
z^{\prime}=h(t) z \text {. }
$$

We can suppose that (4.1) is absolutely convergent for $t=0$, and put

$$
C=\int_{0}^{\infty}|d H(s)|<\infty
$$

For otherwise we can replace $t$ by $t+\epsilon>t$ and the argument below shows that (4.2) is then absolutely convergent for $\operatorname{Re} t>\epsilon$.

From (4.1)-(4.3), we see that $-s d Z(s)=d(H * Z)$, so that

$$
s|d Z(s)| \leqslant d \int_{0}^{s}|H(s-r)||d Z(r)| \text {. }
$$

Hence, by (4.4),

$$
\int_{0}^{s} r|d Z(r)| \leqslant C \int_{0}^{s}|d Z(r)| \text {. }
$$

Consequently, for large $s$,

$$
\int_{s}^{2 s}|d Z(r)| \leqslant(s-C)^{-1} C \int_{0}^{s}|d Z(r)| \leqslant 2 C s^{-1} \int_{0}^{s}|d Z(r)| .
$$

Define $F(s)=\int_{0}^{s}|d Z(r)|$ for $s \geqslant 0$, so that, for large $s, F(2 s) \leqslant\left(1+2 C s^{-1}\right) F(s)$. If $\theta>0$ is arbitrary and $s$ is sufficiently large, then $F(2 s) \leqslant(1+\theta) F(s)$. Hence $F\left(2^{n} s\right) \leqslant(1+\theta)^{n} F(s)$ and so, for large $r, F(r) \leqslant\left(\right.$ const) $r^{\log (1+\theta) / \log 2}$. It follows that

$$
\int_{0}^{\infty} e^{-r t}|d Z(r)|=\int_{0}^{\infty} e^{-r t} d F(r)<\infty \text { for } t>0 .
$$

This completes the proof.

5. Preparations for an induction. There is no loss of generality in assuming that $\lambda(1)=\cdots=\lambda\left(K_{1}\right)=0$ for the change of variables

$$
y=e^{\lambda(1) t_{z}}
$$

transiorms (3.1) to

$$
z^{\prime}=[J-\lambda(1) I+g(t)] z,
$$

which is the same type as (2.1) with $\lambda(k)$ replaced by $\lambda(k)-\lambda(1)$. The following result is contained in Theorem 1.1 of [2]:

Proposition 5.1. Let Assumption (A) hold with $\lambda(1)=0$. Let $\epsilon(k) \geqslant$ 0 for $k=1, \cdots, M$ and $\epsilon(k, k)=\epsilon(k)+\kappa$ or $\epsilon(k, k)=\epsilon(k)$ according as $1 \leqslant k \leqslant K_{1}$ or $K_{1}<k \leqslant M$. Suppose that

$$
\int_{+0} s^{-\max [0, \epsilon(k, k+1)-\epsilon(m, \mu)]}\left|d G_{k \mu}^{k m}(s)\right|<\infty \text { for all } k, k, m, \mu,
$$


and that, for some $j, \quad 1 \leqslant j \leqslant K_{1}$,

$$
\int_{+0} s^{-\epsilon(k, \kappa+1)}\left|d G_{k 0}^{\kappa j}(s)\right|<\infty \text { for all } k, \kappa .
$$

Then (3.1) has a solution $y=y^{j}(t)$

$$
y^{j}(t)=1_{0}^{j}+w_{0}^{j}(t)
$$

where the $N$-vector $w_{0}^{j}$ has the $(k, \kappa)$ th component

$$
w_{k 0}^{\kappa j}(t)=\int_{0}^{\infty} e^{-s t} d W_{k 0}^{k j}(s), \quad \int_{+0} s^{-\epsilon(k, \kappa)}\left|d W_{k 0}^{k j}(s)\right|<\infty
$$

for all $(k, \kappa)$, i.e., $k=1, \cdots, M$ and $\kappa=0, \cdots, e(k)-1$.

For any $N$-vector $y$, let $\bar{y}=\left(y_{(1)}, y_{(2)}\right)$ be an $N$-vector with components obtained by a permutation of those of $y$ as follows: $y_{(1)}=\left(y_{1}^{0}, \cdots, y_{K_{1}}^{0}\right)$ is a $K_{1}$-tuple consisting of the $(k, 0)$ th components of $y$ for $k=1, \cdots, K_{1}$ and $y_{(2)}$ is an $\left(N-K_{1}\right)$-tuple consisting of the other components of $y$. The $(k, \kappa)$ th component $y_{(2) k}^{k}$ of $y_{(2)}$, where $k=1, \cdots, M$ and $k=0, \cdots, \bar{e}(k)-1$ is $y_{(2) k}^{\kappa}=y_{k}^{\bar{\kappa}}$ where $\bar{\kappa}=\kappa+1$ or $\bar{\kappa}=\kappa$ and $\bar{e}(k)=e(k)-1$ or $\bar{e}(k)=e(k)$ according as $k \leqslant K_{1}$ or $k>K_{1}$. We also write $(m, \bar{\mu})$ with $\bar{\mu}=\mu+1$ or $\bar{\mu}=\mu$ according as $m \leqslant K_{1}$ or $m>K_{1}$.

If $e(k)=1$ for some $k \leqslant K_{1}$, then there are no components $y_{(2) k}^{\kappa}$ of $y_{(2)}$ and below all such indices $(k, \kappa)$ should be ignored.

Under the linear change of variables $y \rightarrow \bar{y},(2.4)$ becomes

$$
\begin{aligned}
y_{(1) j}^{\prime}= & \lambda(j) y_{(1) j}+\sum_{m=1}^{K_{1}} g_{j 0}^{0 m} y_{(1) m} \\
& +\left(1-\delta_{1, e(j)}\right) y_{(2) j}^{0}+\sum_{m=1}^{M} \sum_{\mu=0}^{\bar{e}(m)-1} g_{\bar{\mu}}^{0 m} y_{(2) m}^{\mu}, \\
y_{(2) k}^{\kappa^{\prime}}= & \sum_{m=1}^{K_{1}} g_{k 0}^{\bar{k} m} y_{(1) m}+\lambda(k) y_{(2) k}^{\kappa} \\
& +\left(1-\delta_{\kappa, e(k)}\right) y_{(2) k}^{\kappa+1}+\sum_{m=1}^{M} \sum_{\mu=0}^{\bar{e}(m)-1} g_{k \bar{\mu}}^{\bar{k} m} y_{(2) m}^{\mu},
\end{aligned}
$$

where $j=1, \cdots, K_{1}$ in (5.7) and $k=1, \cdots, M$ and $\kappa=0, \cdots, \bar{e}(k)-1$ in (5.8). Also $y_{(2) k}^{k+1}=0$ if $k=\bar{e}(k)-1$, and $\delta_{j k}$ is 1 or 0 according as $j=k$ or $j \neq k$. For brevity, we can write (5.7), (5.8) in vector-matrix notation 


$$
\begin{aligned}
& y_{(1)}^{\prime}=\left[\lambda(1) I_{K_{1}}+g_{11}(t)\right] y_{(1)}+\left[J_{12}+g_{12}(t)\right] y_{(2)}, \\
& y_{(2)}^{\prime}=g_{21}(t) y_{(1)}+\left[\bar{J}+g_{22}(t)\right] y_{(2)} .
\end{aligned}
$$

Here $I_{K_{1}}$ is the unit $K_{1}$-matrix, the $j$ th component of $J_{12} y_{(2)}$ is $\left(1-\delta_{1, e(j)}\right) y_{(2) j}^{0}$ for $j=1, \cdots, K_{1}$, and

$$
\bar{J}=\operatorname{diag}[J(\lambda(1), \bar{e}(1)), \cdots, J(\lambda(M), \bar{e}(M))]
$$

with $J(\lambda(k), \bar{e}(k))$ absent if $\bar{e}(k)=0$.

If $\lambda(1)=0$ and Proposition 5.1 is applicable for $j=1, \cdots, K_{1}$, then the $K_{1}$ solutions $y^{1}, \cdots, y^{K_{1}}$ can be used to reduce the order $N$ of the system (5.7)-(5.8). Let

$$
Z(t)=\left(\begin{array}{ll}
Y_{11}(t) & 0 \\
Y_{21}(t) & I_{N-K_{1}}
\end{array}\right)
$$

be a square $N$-matrix, in which the first $K_{1}$ columns are the $N$-vector solutions $y^{1}, \cdots, y^{K_{1}}$. Thus, $Y_{11}$ is the square $K_{1}$-matrix

$$
Y_{11}(t)=I_{K_{1}}+\left(w_{k 0}^{0 j}(t)\right) \text { for } j, k=1, \cdots, K_{1},
$$

and $Y_{21}$ is the rectangular matrix

$$
Y_{21}(t)=\left(w_{k 0}^{\bar{\kappa} j}(t)\right) \quad \text { for } j=1, \cdots, K_{1},
$$

$k=1, \cdots, M$ and $\kappa=0, \cdots, \bar{e}(k)-1$. In (5.11) [or (5.12)], $j$ is the column index and $k$ [or $(k, \kappa)$ ] is the row index. It is clear that $\operatorname{det} Z=\operatorname{det} Y_{11} \neq 0$ for large $\operatorname{Re} t$. For such $t$, write the inverse of (5.10) as

$$
Z^{-1}(t)=\left(\begin{array}{ll}
Y_{11}^{-1}(t) & 0 \\
Z_{21}(t) & I_{N-K_{1}}
\end{array}\right), \quad Z_{21}=-Y_{21} Y_{11}^{-1}
$$

and $Y_{11}^{-1}$ is of the form

$$
Y_{11}^{-1}(t)=I_{K_{1}}+\left(w_{j}^{k}(t)\right) \text { for } j, k=1, \cdots, K_{1},
$$

where $\bar{w}_{j}^{k}$ is a Laplace-Stieltjes transform.

Introduce the change of variables $\bar{y}=Z(t) \bar{z}$, that is, $\bar{z}=\left(z_{(1)}, z_{(2)}\right)$ and

$$
y_{(1)}=Y_{11}(t) z_{(1)}, \quad y_{(2)}=Y_{21}(t) z_{(1)}+z_{(2)} .
$$

Then (5.9) becomes

$$
z_{(1)}^{\prime}=Y_{11}^{-1}\left[J_{12}+g_{12}\right] z_{(2)},
$$


(5.17) $z_{(2)}^{\prime}=\left\{Z_{21}\left[J_{12}+g_{12}\right]+\bar{J}+g_{22}\right\} z_{(2)} \equiv[\bar{J}+\bar{g}(t)] z_{(2)}$;

cf. [4, p. 50]. The matrix $\bar{g}(t)$ is defined by (5.17): The system (5.17) is of order $N-K_{1}$, while (5.16) can be solved by a quadrature if a solution $z_{(2)}$ of (5.17) is known.

Proposition 5.2. Let Assumption (A) hold with $\lambda(1)=0$. Let $e(k) \geqslant 0$ and $\epsilon(k, \kappa)$ be defined as in Proposition 5.1. Assume that

$$
\int_{+0} s^{-\epsilon(k, k+1)}\left|d G_{k \mu}^{\kappa m}(s)\right|<\infty
$$

for $k, m=1, \cdots, M$ and $\kappa[$ or $\mu]=0, \cdots, e(k)-1$ [or $e(m)-1]$. Then Proposition 5.1 is applicable with $j=1, \cdots, K_{1}$ and the entries in the matrix $\bar{g}(t)=\left(\bar{g}_{k \mu} \mathrm{km}\right)$ of (5.17) are Laplace-Stieltjes transforms such that

$$
\int_{+0} s^{-\epsilon(k, \kappa+1)}\left|d \bar{G}_{k \mu}^{k m}(s)\right|<\infty
$$

for $k=1, \cdots, M$ and $\kappa[$ or $\mu]=0, \cdots, \bar{e}(k)-1$ [or $\bar{e}(m)-1]$.

Proof. In order to verify the applicability of Proposition 5.1 for $j=$ $1, \cdots, K_{1}$, note that (5.3)-(5.4) follow from (5.18). In view of the equivalence of (5.7)-(5.8) and (5.9) and in view of (5.12)-(5.14), we can write the entry $\bar{g}_{k \mu}^{k m}$ of $\bar{g}$ in (5.17) as

$$
\bar{g}_{k \mu}^{\kappa m}=\sum_{j=0}^{K_{1}} w_{k 0}^{\bar{\kappa} j}\left[c_{j k \mu}^{k m}+u_{j k \mu}^{\kappa m}\right]+g_{k \bar{\mu}}^{\bar{\kappa}},
$$

where $c_{j k \mu}^{k m}$ is a constant and $u_{j k \mu}^{k m}$ is a Laplace-Stieltjes transform.

Note that $\epsilon(k, \bar{\kappa})=\epsilon(k, \kappa+1)$ for all $(k, \kappa)$, since $\bar{\kappa}=\kappa+1$ if $k \leqslant K_{1}$ and $\epsilon(k, \kappa)$ is independent of $\kappa$ for $k>K_{1}$. By $(5.6), \epsilon(k, \bar{\kappa})=\epsilon(k, \kappa+1)$ is admissible for $w_{k 0}^{\bar{k} j}$ and, by $(5.18), \epsilon(k, \bar{k}+1) \geqslant \epsilon(k, \kappa+1)$ is admissible for $g_{k \mu}^{\bar{k} m}$. Hence (5.19) follows from Proposition 3.1.

The following proposition will not be used below but can be used to obtain generalizations of Theorem 2.1.

Proposition 5.3. Let Assumption (A) hold with $\lambda(1)=0$. Let $\epsilon(k) \geqslant 0$ and $\epsilon(k, \kappa)$ be defined as in Proposition 5.1. Assume that

(5.22) $\int_{+0} s^{-\max [0, \epsilon(k, \kappa+1)-\epsilon(m)]}\left|d G_{k \mu}^{\kappa m}(s)\right|<\infty \quad$ for $1 \leqslant k \leqslant K_{1}<m \leqslant M$,

$$
\int_{+0} s^{-\epsilon(k)}\left|d G_{k \mu}^{k m}(s)\right|<\infty \quad \text { for } K_{1}<k \leqslant M, \quad 1 \leqslant m \leqslant M,
$$


and $k$ [or $\mu]=0, \cdots, e(k)-1$ [or $e(m)-1]$. Then Proposition 5.1 is applicable with $j=1, \cdots, K_{1}$ and the entries in the matrix $\bar{g}(t)=\left(\bar{g}_{k \mu}^{k m}\right)$ of (5.17) are Laplace-Stieltjes transforms for $k, m=1, \cdots, M$ and $\kappa[$ or $\mu]=$ $0, \cdots, \bar{e}(k)-1$ [or $\bar{e}(m)-1]$ and (5.21)-(5.23) hold if $G_{k \mu}^{k m}$ is replaced by $\bar{G}_{k \mu}^{k m}$.

We omit the proof which is similar to that of Proposition 5.2.

6. Proof of Theorem 2.1. The proof is by induction on the order $N$ of the system (2.1). The result is easy if $N=1$ (and is contained in [2]). Assume that $N>1$ and the validity of Theorem 2.1 and the remark following it for a system of order less than $N$. Assume the conditions of Theorem 2.1 with (2.12) replaced by (2.18) for $k=1, \cdots, K_{1}$. Without loss of generality, it can be supposed that $\lambda(1)=0$ (so that $\lambda(k)$ is real and nonnegative for $1 \leqslant k \leqslant$ $K$ and, in fact, positive for $\left.K_{1}<k \leqslant K\right)$. Since the case $i(n)=1$ is contained in Theorem 1.1 of [2], we shall suppose that $i(n)>1$ (i.e., $n>K_{1}$ ). Furthermore, in view of Corollary 2.2, we shall consider only large $\operatorname{Re} t$ (and it is implicitly assumed that all Laplace-Stieltjes transforms below are absolutely convergent on some half-plane $\operatorname{Re} t \geqslant$ const).

Let $\epsilon(k)=\gamma(k)-e(k)$ for $1 \leqslant k \leqslant K_{1}$ and $\epsilon(k)=\gamma(k)$ for $K_{1}<k \leqslant$ $M$. Then Proposition 5.2 is applicable to the system (2.1) and leads to a system (5.17) of order $N-K_{1}$. This system satisfies the analogue of the conditions of Theorem 2.1 with (2.12) replaced by (2.18) for $1 \leqslant k \leqslant K_{1}$. Hence, by the induction hypothesis, the conclusions of Theorem 2.1 hold for the system (5.17). Let a solution corresponding to (2.13) be

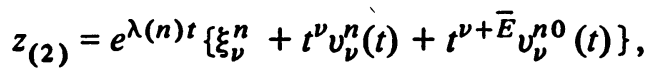

where the $(k, \kappa)$ th components $v_{k \nu}^{k n}, v_{k \nu}^{k n 0}$ of the $N-K_{1}$ vectors $v_{\nu}^{n}, v_{\nu}^{n 0}$ are Laplace-Stieltjes transforms of the form

$$
\begin{gathered}
v_{k \nu}^{k n}(t)=\int_{0}^{\delta^{\prime}} e^{-s t} d V_{k \nu}^{k n}(s), \quad v_{k \nu}^{k n 0}(t)=\int_{\delta^{\prime}}^{\infty} e^{-s t} d V_{k \nu}^{k n 0}(s), \\
\int_{+0} s^{-\gamma(k)+e(k)}\left|d V_{k \nu}^{k n}(s)\right|<\infty \quad \text { for } 1 \leqslant k \leqslant K_{i(n)-1}, \\
\int_{+0} s^{-\gamma(k)+e(k)-\kappa}\left|d V_{k \nu}^{k n}(s)\right|<\infty \quad \text { for } K_{i(n)-1}<k \leqslant K_{i(n)}, \\
\int_{+0} s^{-\gamma(k)}\left|d V_{k \nu}^{k n}(s)\right|<\infty \text { for } K_{i(n)}<k \leqslant M .
\end{gathered}
$$

Here $0<\delta^{\prime}<\min \left[\lambda\left(K_{j}\right)-\lambda\left(K_{j}-1\right)\right]$ for $j=2, \cdots, i(n)$ [or $j=3, \cdots, i(n)$ ] if $e\left(K_{1}\right)>1$ [or $e\left(K_{1}\right)=1$ ]. Also $\bar{E}=E(i(n)-1)-1$ since $\bar{e}(k)=e(k)-1$ for $k=1, \cdots, K_{1}$. 
In view of (5.14) and (5.16), $z_{(1) j}^{\prime}$ is $e^{\lambda(n) t}$ times

$$
\begin{aligned}
& \sum_{k=1}^{K_{1}}\left(\delta_{j k}+\bar{w}_{j}^{k}\right)\left\{\left(1-\delta_{1, e(k)}\right)\left(t^{\nu} v_{k \nu}^{0 n}+t^{\nu+\bar{E}_{v}} v_{k \nu} 0\right)\right. \\
& \left.+\sum_{m=1}^{M} \sum_{\mu=0}^{\bar{e}(m)-1} g_{k \bar{\mu}}^{0 n}\left(\xi_{m \nu}^{\mu n}+t^{\nu} v_{m \nu}^{\mu n}+t^{\nu+\bar{E}_{v}}{ }_{m \nu}^{\mu n 0}\right)\right\} .
\end{aligned}
$$

Since $\gamma(k)-e(k)$ is admissible for the off-diagonal elements $w_{k 0}^{0 j}, j \neq k$, of the $k$ th row of $Y_{11}$, Proposition 3.3 implies that $\gamma(j)-e(j)$ is admissible for $\bar{w}_{j}^{k}=\delta_{j k}+\bar{w}_{j}^{k}$ if $j \neq k$. For the term $k=j$ of the sum over $k$, we use the facts that $\epsilon(j, 1)>\gamma(j)-e(j)$ is admissible for $g_{k \bar{\mu}}^{0 n}, k=j$, and that $\epsilon(j)=$ $\gamma(j)-e(j)$ is admissible for $v_{k \nu}^{0 n}, k=j$, by (6.3). Also $\xi_{m \nu}^{\mu n}$ is 0 if $m \neq n$ or $m=n, \mu>\nu$ and is $t^{\nu-\mu} /(\nu-\mu)$ ! if $m=n, \mu \leqslant \nu$. Thus the last display is of the form $t^{\nu} v+t^{\nu+\bar{E}_{0}} v^{0}$, where $v, v^{0}$ are Laplace-Stieltjes transforms such that $d V(s)=0$ for $s \geqslant \delta^{\prime}, d V^{0}(s)=0$ for $s \leqslant \delta^{\prime}$ and $\gamma(1)-e(1)$ is admissible for $v$. Hence

$$
z_{(1) j}^{\prime}=e^{\lambda(n) t}\left[t^{\nu} v+t^{\left.\nu+\bar{E}_{v^{0}}\right]}\right.
$$

Since $n>K_{1}$ implies that $\lambda(n)=\lambda(n)-\lambda(1)>0$, quadratures and Proposition 3.6 give a solution of (5.16) in which the $j$ th component $z_{(1) j}$ is of the form

$$
z_{(1) j}=e^{\lambda(n) t}\left[t^{\nu} v+t^{\nu+E(i(n)-1)} v^{0}\right]
$$

where $v, v^{0}$ are different functions with analogous properties, but with $d V(s)=$ 0 [or $d V^{0}(s)=0$ ] for $s \geqslant \delta$. [or $s \leqslant \delta$ ] and

$$
0<\delta<\min \left[\delta^{\prime}, \lambda\left(K_{2}\right)-\lambda\left(K_{1}\right), \cdots, \lambda\left(K_{i(n)}\right)-\lambda\left(K_{i(n)-1}\right)\right]
$$

and $E(i(n)-1)=\bar{E}+1$. From (5.14) and (5.15), we obtain an identical type of formula for the $j$ th component of $y_{(1)}$, i.e., for $y_{j}^{0}, j=1, \cdots, K_{1}$. This implies (2.15) for $1 \leqslant k \leqslant K_{1}$ and $\kappa=0$.

From (5.12) and (5.15), we have

$$
y_{k}^{\bar{K}}=y_{(2) k}^{\kappa}=\sum_{j=1}^{K_{1}} w_{k 0}^{\bar{K} j} z_{(1) j}+z_{(2) k}^{\kappa}
$$

for $k=1, \cdots, M$ and $\kappa=0, \cdots, \bar{e}(k)-1$. Since $\epsilon(k, \bar{\kappa})$ is admissible for $w_{k 0}^{\bar{K} j}$, the formulas (6.1)-(6.5) and (6.8) imply the remaining formulas in (2.15)(2.17), i.e., those for $(k, \kappa)$ with either $\kappa \neq 0$ or $k>K_{1}$. This completes the proof.

7. On Corollary 2.1. The proof is by induction on the order $N$ and depends on a re-examination of the arguments of the last section. Corollary 2.1 is valid 
for $N=1$ (in fact the case $1 \leqslant n \leqslant K_{1}$ and $\nu=0$ are contained in Corollary 3.3 of [2]). The cases $1 \leqslant n \leqslant K_{1}$ and arbitrary $\nu=0, \cdots, e(n)-1$ follow from the proof of Theorem 5.2 of [2] in the same way that Corollary 3.3 follows from the proofs of Theorems 1.1 and 1.2.

Assume $N>1$ and the validity of the corollary for systems of order $N-$ 1. We shall consider only the cases $n>K_{1}$.

Assume the hypotheses of part (i) of Corollary 2.1. Then the validity of the corollary for $n=1, \nu=0$ and the formulas (5.12) show that the system (5.11) satisfies the corresponding set of hypotheses. Hence the functions $V_{k \nu}^{k n}(s)$ of (6.1)-(6.2) vanish for $0 \leqslant s<\Delta$. The arguments leading to (6.7) show that if $V$ is the determining function for $v$ in (6.7), then $d V(s)=0$ for $0 \leqslant$ $s<\Delta$. (This depends on the fact that $\xi_{m \nu}^{\mu n}$ in (6.6) is 0 if $m \neq n$ or if $m=$ $n$ and $\mu>\nu$.) The proof of Proposition 3.6 shows that if $V$ belongs to $v$ in (6.8), then $d V(s)=0$ for $0 \leqslant s<\Delta$. We now obtain part (i) of Corollary 2.1 from (5.8), (5.9).

The proofs of parts (ii), (iii) and (iv) are similar and will be omitted.

8. Applications to Bessel and Legendre functions. The change of variables $v=t^{1 / 2} u$ transforms the Bessel differential equation of order $\mu$,

$$
u^{\prime \prime}+u^{\prime} / t+\left(1-\mu^{2} / t^{2}\right) u=0
$$

wito the equation

$$
v^{\prime \prime}+\left(1-\beta / t^{2}\right) v=0, \text { where } \beta=\mu^{2}-1 / 4 .
$$

Let $H_{\mu}^{(1)}(t), H_{\mu}^{(2)}(t)$ be the Hankel functions which are solutions of (8.1) (so that $t^{1 / 2} H_{\mu}^{(1)}, t^{1 / 2} H_{\mu}^{(2)}$ are solutions of (8.2)) satisfying

$$
c_{1} t^{1 / 2} H_{\mu}^{(1)}(t) \sim e^{i t}, \quad c_{2} t^{1 / 2} H_{\mu}^{(2)}(t) \sim e^{-i t}
$$

as $t \rightarrow \infty$, where

$$
c_{j}=(\pi / 2)^{1 / 2} e^{ \pm i(\mu+1 / 2) \pi / 2} \text { and } \pm=(-1)^{j+1} .
$$

It follows from Theorem 1.1 (in fact from Theorem 2.1 of [3]) that the solutions (8.3) of (8.2) have representations in terms of Laplace-Stieltjes transforms absolutely convergent for $\operatorname{Re} t>0$,

$$
c_{j} t^{1 / 2} H_{\mu}^{(j)}(t)=e^{ \pm i t}\left[1+\int_{+0}^{\infty} e^{-s t} d W_{j}(s)\right], \quad \text { where } \pm=(-1)^{j+1}
$$

for $j=1,2$. We shall verify the following:

Proposition 8.1. In the formula (8.5),

$$
W_{j}(s)=P\left(1+(-1)^{j+1} i s\right) \text { for } s \geqslant 0,
$$


where $P=P_{\nu}(\sigma), \nu=\mu-1 / 2$, is the unique solution of the Legendre differential equation

$$
d\left[\left(1-\sigma^{2}\right) d P / d \sigma\right] / d \sigma+\nu(\nu+1) P=0,
$$

regular at $\sigma=1$ and normalized by $P(1)=1$.

In (8.5) and (8.6), we have $W_{j}(0)=w_{j}(+0)=1$ (instead of our usual normalization $\left.W_{j}(0)=W_{j}(+0)=0\right)$ so that, in any case, $s=0$ does not contribute to the integral in (8.5). The formulas (8.5), (8.6) are analogous to, but different from, results of Whittaker [6] (cf. [5, pp. 173-175]).

Proof. For the sake of convenience, consider the case $j=1$. Since the left side of (8.5) is a solution of (8.2), it follows that if $w=w_{j}(t)$ is the Laplace-Stieltjes transform in (8.5), then

$$
w^{\prime \prime}+2 i w^{\prime}-\beta / t^{2}-\beta w / t^{2}=0 .
$$

By Corollary 2.1 of $[3], W=W_{1}(s)$ is of class $C^{\infty}$ for $s>0$, so that

implies that

$$
1 / t^{2}=\int_{0}^{\infty} e^{-s t} s d s
$$

$$
\left(s^{2}-2 i s\right) W^{\prime}-\beta s-\beta \int_{0}^{s}(s-r) W^{\prime}(r) d r=0 .
$$

Differentiating this relation gives

$$
\left[\left(s^{2}-2 i s\right) W^{\prime}\right]^{\prime}-\beta-\beta \int_{0}^{s} W^{\prime}(r) d r=0 .
$$

Hence if $W$ is normalized by $W(0)=1$, equation (8.8) can be written as

$$
\left[\left(s^{2}-2 i s\right) W^{\prime}\right]^{\prime}-\beta W=0 .
$$

The point $s=0$ is a regular singular point for (8.9) with the indicial exponents 0 and 0 , so that there exists a unique solution $W(s)$ (up to a constant factor) such that $W^{\prime}(s)$ is of class $L^{1}$ on $0<s \leqslant \epsilon$. This solution is regular at $s=0$ and is uniquely determined by the normalization $W(0)=1$. The change of variables $\sigma=1+i$ is, $W=P$ reduces (8.9) to the Legendre equation (8.7) with $\nu=\mu-1 / 2$. This completes the proof.

In view of the relations, $H_{\mu}^{(1)}=J_{\mu}+i Y_{\mu}$ and $H_{\mu}^{(2)}=J_{\mu}-i Y_{\mu}$, we have $H_{\mu}^{(1)}(t) H_{\mu}^{(2)}(t)=J_{\mu}^{2}+Y_{\mu}^{2}$. Hence, by $(8.5)$,

$$
\pi t\left(J_{\mu}^{2}+Y_{\mu}^{2}\right) / 2=\left[1+\int_{0}^{\infty} e^{-s t} d W_{1}(s)\right]\left[1+\int_{0}^{\infty} e^{-s t} d W_{2}(s)\right],
$$

which can be written in the form

$$
\pi t\left(J_{\mu}^{2}+Y_{\mu}^{2}\right) / 2=1+\int_{0}^{\infty} e^{-s t} d Q(s)
$$


where, if $Q$ is normalized by $Q(0)=Q(+0)=1$,

$$
Q(s)=W_{1}(s)+\int_{0}^{s} W_{1}(s-r) d W_{2}(r) \text { for } s \geqslant 0 .
$$

If this is compared with the relation

$$
\pi t\left(J_{\mu}^{2}+Y_{\mu}^{2}\right) / 2=1+\int_{0}^{\infty} e^{-s t} d P\left(1+s^{2} / 2\right) \text {, with } P=P_{\mu-1 / 2}(\sigma),
$$

proved in $\S 11$ of [3], we obtain the following (apparently new) functional relationship for $P=P_{\nu}$ :

Proposition 8 .2. For arbitrary $\nu$ and real $s \geq 0$, the Legendre function $P=P_{\nu}(\sigma)$ satisfies

$$
P\left(1+s^{2} / 2\right)=P(1+i s)-i \int_{0}^{s} P(1+i(s-r)) P^{\prime}(1-i r) d r,
$$

where $P^{\prime}(1-i r)=[d P(\sigma) / d \sigma]_{\sigma=1-i r}$.

We can obtain other functional equations in a similar manner. If $v_{1}, v_{2}$ is any pair of solutions of (8.2), then the product $v=v_{1} v_{2}$ is a solution of

$$
v^{\prime \prime \prime}+4\left(1-\beta / t^{2}\right) v^{\prime}+\left(4 \beta / t^{3}\right) v=0 ;
$$

Appell [1]. This is a perturbation of the equation $v^{\prime \prime \prime}+4 v^{\prime}=0$ with constant coefficients having characteristic numbers $0, \pm 2 i$. Hence by Theorem 2.1 of [3], it has solutions representable in the form

$$
\left[c_{j} t^{1 / 2} H_{\mu}^{(j)}(t)\right]^{2}=e^{ \pm 2 i t}\left[1+\int_{+0}^{\infty} e^{-s t} d U_{j}(s)\right], \text { where } \pm=(-1)^{j+1},
$$

and the solution (8.10). Arguing as in the proof of Proposition 8.1, we see that if $u=u_{j}(t)$ denotes the Laplace-Stieltjes transform in the last formula, then

$$
\begin{aligned}
& u^{\prime \prime \prime} \pm 6 i u^{\prime \prime}-8 u^{\prime}-4 \beta u^{\prime} / t^{2} \\
& +\left[4 \beta / t^{3} \mp 8 \beta i / t^{2}\right] u+4 \beta / t^{3} \mp 8 \beta i / t^{2}=0 .
\end{aligned}
$$

Thus if $U=U_{j}(s)$, then

$$
-s\left(s^{2} \mp 6 i s-8\right) U^{\prime}+2 \beta \int_{0}^{s}\left[\left(s^{2}-r^{2}\right) \mp 4 i(s-r)\right] U^{\prime}(r) d r+2 \beta s^{2} \mp 8 \beta i s=0 .
$$

Differentiating this relation gives

$$
\text { - }\left[s(s \mp 4 i)(s \mp 2 i) U^{\prime}\right]^{\prime}+4 \beta(s \mp 2 i) U=0 \text { if } U(0)=1 .
$$

Introduce the new variable $\sigma=-1-(s \mp 2 i)^{2} / 2=1-s^{2} / 2 \pm 2 i s$ and $U=P$ to obtain (8.7). Hence

$$
U=U_{j}(s)=P\left[-1-(s \mp 2 i)^{2} / 2\right], \text { where } \mp=(-1)^{j} .
$$

From (8.6), (8.13) and (8.14), we get 
PROPOSITION 8.3. For arbitrary $\nu$ and (real) $s \geqslant 0$, the Legendre function $P=P_{\nu}(\sigma)$ satisfies the functional equation

$$
P\left[-1-(s \mp 2 i)^{2} / 2\right]=P(1 \pm i s) \pm i \int_{0}^{s} P[1 \pm i(s-r)] P^{\prime}(1 \pm i r) d r,
$$

where $P^{\prime}(1 \pm i r)=[d P / d \sigma]_{\sigma=1 \pm i r}$.

\section{REFERENCES}

1. P. Appell, Sur les transformations des équations différentielles linéaires, C. R. Acad. Sci. Paris 91 (1880), 211-214.

2. J. D'Archangelo, Linear ordinary differential equations with Laplace-Stielties transforms as coefficients, Trans. Amer. Math. Soc. 195 (1974), 115-145.

3. P. Hartman, On differential equatior
$Y_{\mu}^{2}$, Amer. J. Math. 95 (1973), 553-593.

4. - Ordinary differential equations, Baltimore, Md., 1973.

5. G. N. Watson, $A$ treatise on the theory of Bessel functions, 2 nd ed., Cambridge Univ. Press, Cambridge; Macmillan, New York, 1944. MR 6, 64.

6. E. T. Whittaker, On a new connexion of Bessel functions with Legendre functions, Proc. London Math. Soc. 35 (1903), 198-206.

7. A Wintner, On the existence of Laplace solutions for linear differential equations of second order, Amer. J. Math. 72 (1950), 442-450. MR 12, 179; 1002.

8. - On the small divisors in integrations by Laplace transforms, Amer. J. Math. 73 (1951), 173-180. MR 13, 37.

9. - Ordinary differential equations and Laplace transforms, Amer. J. Math. 79 (1957), 265-294. MR 19, 271.

DEPARTMENT OF MATHEMATICS, UNITED STATES NAVAL ACADEMY, ANNAP. OLIS, MARYLAND 21218

DEPARTMENT OF MATHEMATICS, JOHNS HOPKINS UNIVERSITY, BALTIMORE, MARYLAND 21218 